\title{
Study of the $B_{c} \rightarrow J / \psi+D_{q}^{(*)}$ decays in covariant confined quark model
}

\author{
Aidos Issadykov ${ }^{1,2, \star}$ \\ ${ }^{1}$ Joint Institute for Nuclear Research,141980 Dubna, Russia \\ ${ }^{2}$ The Institute of Nuclear Physics,Ministry of Energy of the Republic of Kazakhstan, 050032 Almaty, Kaza- \\ khstan
}

\begin{abstract}
In this work we study the $B_{c} \rightarrow J / \psi+D_{q}^{(*)}$ decays. It was defined ratios $\left(R_{D_{s}^{*} / D_{s}}, R_{D^{*} / D}, R_{D^{*} / D_{s}^{*}}\right.$ and $\left.R_{D / D_{s}}\right)$ of nonleptonic branching ratios of $B_{c}$ meson, which will be hopefully tested on LHC experiments. We compare the obtained results with available experimental data and with the results from other theoretical approaches.
\end{abstract}

\section{Introduction}

Recently the ATLAS Collaboration reported on the measurement of the various branching fractions of the decays $B_{c}^{+} \rightarrow J / \psi D_{s}^{+}$and $B_{c}^{+} \rightarrow J / \psi D_{s}^{*+}$ [1]. The first observations of these decays have been performed by the LHCb Collaboration [2]. In view of these developments, we decided to calculate ratios of branching fractions within the covariant confined quark model(CCQM).

The decay properties of the above processes were studied in various theoretical approaches [3-12].

\section{Effective Hamiltonian and matrix element}

The effective Hamiltonian describing the $B_{c}$ nonleptonic decays into charmonium and $D\left(D_{s}\right)$ meson is given by (see, Ref. [13])

$$
\begin{array}{rlrl}
\mathcal{H}_{\mathrm{eff}} & =-\frac{G_{F}}{\sqrt{2}} V_{c b} V_{c q}^{\dagger} \sum_{i=1}^{6} C_{i} O_{i}, & \\
O_{1} & =\left(\bar{c}_{a_{1}} b_{a_{2}}\right)_{V-A}\left(\bar{q}_{a_{2}} c_{a_{1}}\right)_{V-A}, & & O_{2}=\left(\bar{c}_{a_{1}} b_{a_{1}}\right)_{V-A},\left(\bar{q}_{a_{2}} c_{a_{2}}\right)_{V-A}, \\
O_{3} & =\left(\bar{q}_{a_{1}} b_{a_{1}}\right)_{V-A}\left(\bar{c}_{a_{2}} c_{a_{2}}\right)_{V-A}, & O_{4}=\left(\bar{q}_{a_{1}} b_{a_{2}}\right)_{V-A}\left(\bar{c}_{a_{2}} c_{a_{1}}\right)_{V-A}, \\
O_{5} & =\left(\bar{q}_{a_{1}} b_{a_{1}}\right)_{V-A}\left(\bar{c}_{a_{2}} c_{a_{2}}\right)_{V+A}, & O_{6}=\left(\bar{q}_{a_{1}} b_{a_{2}}\right)_{V-A}\left(\bar{c}_{a_{2}} c_{a_{1}}\right)_{V+A},
\end{array}
$$

where the subscript $V-A$ refers to the usual left-chiral current $O^{\mu}=\gamma^{\mu}\left(1-\gamma^{5}\right)$ and $V+A$ to the usual right-chiral one $O_{+}^{\mu}=\gamma^{\mu}\left(1+\gamma^{5}\right)$. The $a_{i}$ denote the color indices. The quark $q$ stands for either $s$ or $d$. The numerical values of the Wilson coefficients are taken as in Ref.[14]. They are listed in Table 1. Since in NNLO the numerical values of the $C_{5}$ and $C_{6}$ coefficients are less to the order than the $C_{3}$

^e-mail: issadykov@jinr.ru 
Table 1. Values of the Wilson coefficients in NNLO.

\begin{tabular}{cccccc}
\hline$C_{1}$ & $C_{2}$ & $C_{3}$ & $C_{4}$ & $C_{5}$ & $C_{6}$ \\
\hline-0.2632 & 1.0111 & -0.0055 & -0.0806 & 0.0004 & 0.0009 \\
\hline
\end{tabular}

and $C_{4}$ coefficients respectively, we drop the contribution from those operators.

By using the Fierz transformation one can check that $O_{3}=O_{1}$ and $O_{4}=O_{2}$. Then the calculation of the matrix elements describing the nonleptonic decays of the $B_{c}$ meson into charmonium and $D\left(D_{s}\right)$ meson is straightforward. The combinations of the Wilson coefficients appear as $a_{1}=C_{2}+C_{4}+\xi\left(C_{1}+\right.$ $\left.C_{3}\right)$ and $a_{2}=C_{1}+C_{3}+\xi\left(C_{2}+C_{4}\right)$ with $\xi=1 / N_{c}$. In the numerical calculations we set the colorsuppressed parameter $\xi$ to zero. Then the Wilson coefficients are equal to

$$
a_{1}=C_{2}+C_{4}=0.93, \quad \text { and } \quad a_{2}=C_{1}+C_{3}=-0.27
$$

which should be compared with the old ones $a_{1}=1.14$ and $a_{2}=-0.20$ used in paper [3].

\section{Invariant and helicity amplitudes}

The invariant form factors for the semileptonic $B_{c}$ decay into the hadron with spin $S=0,1$ are defined by

$$
\begin{aligned}
\mathcal{M}_{S=0}^{\mu}= & P^{\mu} F_{+}\left(q^{2}\right)+q^{\mu} F_{-}\left(q^{2}\right), \\
\mathcal{M}_{S=1}^{\mu}= & \frac{1}{m_{1}+m_{2}} \epsilon_{v}^{\dagger}\left\{-g^{\mu v} P q A_{0}\left(q^{2}\right)+P^{\mu} P^{v} A_{+}\left(q^{2}\right)+q^{\mu} P^{v} A_{-}\left(q^{2}\right)\right. \\
& \left.\quad+i \varepsilon^{\mu v \alpha \beta} P_{\alpha} q_{\beta} V\left(q^{2}\right)\right\},
\end{aligned}
$$

where $P=p_{1}+p_{2}$ and $q=p_{1}-p_{2}$. Here $p_{1}$ is the momentum of the ingoing meson with a mass $m_{1}\left(B_{c}\right)$ and $p_{2}$ is the momentum of the outgoing meson with a mass $m_{2}$. It is convenient to express all physical observables through the helicity form factors $H_{m}$. The helicity form factors $H_{m}$ can be written in terms of the invariant form factors in the following way [15]:

Spin $\mathrm{S}=0$ :

$$
H_{t}=\frac{1}{\sqrt{q^{2}}}\left\{\left(m_{1}^{2}-m_{2}^{2}\right) F_{+}+q^{2} F_{-}\right\}, \quad H_{ \pm}=0, \quad H_{0}=\frac{2 m_{1}\left|\mathbf{p}_{2}\right|}{\sqrt{q^{2}}} F_{+} .
$$

Spin $S=1$ :

$$
\begin{aligned}
H_{t} & =\frac{1}{m_{1}+m_{2}} \frac{m_{1}\left|\mathbf{p}_{2}\right|}{m_{2} \sqrt{q^{2}}}\left\{\left(m_{1}^{2}-m_{2}^{2}\right)\left(A_{+}-A_{0}\right)+q^{2} A_{-}\right\}, \\
H_{ \pm} & =\frac{1}{m_{1}+m_{2}}\left\{-\left(m_{1}^{2}-m_{2}^{2}\right) A_{0} \pm 2 m_{1}\left|\mathbf{p}_{2}\right| V\right\}, \\
H_{0} & =\frac{1}{m_{1}+m_{2}} \frac{1}{2 m_{2} \sqrt{q^{2}}}\left\{-\left(m_{1}^{2}-m_{2}^{2}\right)\left(m_{1}^{2}-m_{2}^{2}-q^{2}\right) A_{0}+4 m_{1}^{2}\left|\mathbf{p}_{2}\right|^{2} A_{+}\right\} .
\end{aligned}
$$

Here $\left|\mathbf{p}_{\mathbf{2}}\right|=\lambda^{1 / 2}\left(m_{1}^{2}, m_{2}^{2}, q^{2}\right) /\left(2 m_{1}\right)$ is the momentum of the outgoing meson in the $B_{c}$ rest frame. 
The nonleptonic $B_{c}$ decay widths in terms of the helicity amplitudes are given by

$$
\begin{aligned}
\Gamma\left(B_{c}^{+} \rightarrow J / \psi D_{q}^{+}\right) & =N_{W}\left\{-a_{1} f_{D_{q}} m_{D_{q}} H_{t}^{B_{c} \rightarrow J / \psi}\left(m_{D_{q}}^{2}\right)+a_{2} f_{J / \psi} m_{J / \psi} H_{0}^{B_{c} \rightarrow D_{q}}\left(m_{J / \psi}^{2}\right)\right\}^{2}, \\
\Gamma\left(B_{c}^{+} \rightarrow J / \psi D_{q}^{*+}\right) & =N_{W} \sum_{i=0, \pm}\left\{a_{1} f_{D^{*}} m_{D_{q}^{*}} H_{i}^{B_{c} \rightarrow J / \psi}\left(m_{D_{q}^{*}}^{2}\right)+a_{2} f_{J / \psi} m_{J / \psi} H_{i}^{B_{c} \rightarrow D_{q}^{*}}\left(m_{J / \psi}^{2}\right)\right\}^{2},
\end{aligned}
$$

where we use the short notation

$$
N_{W} \equiv \frac{G_{F}^{2}}{16 \pi} \frac{\left|\mathbf{p}_{2}\right|}{m_{1}^{2}}\left|V_{c b} V_{c q}^{\dagger}\right|^{2}
$$

\section{Numerical results}

All necessary details and values of the calculations of the leptonic decay constants and hadronic form factors may be found in recent publications $[14,16]$. In Table 2 we show widths of the $B_{c}$ meson for general values of the Wilson coefficients $a_{1}$ and $a_{2}$ obtained in this work and in other works.

Table 2. Exclusive nonleptonic decay widths of the $B_{c}$ meson in units of $10^{-15} \mathrm{GeV}$ for general values of the Wilson coefficients $a_{1}$ and $a_{2}$.

\begin{tabular}{|l|c|c|c|c|}
\hline & $B_{c}^{+} \rightarrow J / \psi D_{s}^{+}$ & $B_{c}^{+} \rightarrow J / \psi D_{s}^{*+}$ & $B_{c}^{+} \rightarrow J / \psi D^{+}$ & $B_{c}^{+} \rightarrow J / \psi D^{*+}$ \\
\hline CCQM & $\left(-1.84 a_{1}+2.47 a_{2}\right)^{2}$ & $\left(3.26 a_{1}+2.74 a_{2}\right)^{2}$ & $\left(-0.34 a_{1}+0.36 a_{2}\right)^{2}$ & $\left(0.66 a_{1}+0.51 a_{2}\right)^{2}$ \\
{$[3]$} & $\left(2.19 a_{1}+1.32 a_{2}\right)^{2}$ & $\left(3.69 a_{1}+2.35 a_{2}\right)^{2}$ & $\left(0.462 a_{1}+0.277 a_{2}\right)^{2}$ & $\left(0.785 a_{1}+0.460 a_{2}\right)^{2}$ \\
{$[4]$} & $\left(1.02 a_{1}+1.95 a_{2}\right)^{2}$ & & $\left(0.177 a_{1}+0.442 a_{2}\right)^{2}$ & \\
{$[5]$} & $\left(0.67 a_{1}+0.23 a_{2}\right)^{2}$ & $\left(1.1 a_{1}+1.04 a_{2}\right)^{2}$ & $\left(0.13 a_{1}+0.047 a_{2}\right)^{2}$ & $\left(0.20 a_{1}+0.23 a_{2}\right)^{2}$ \\
{$[17]$} & $\left(1.65 a_{1}+2.92 a_{2}\right)^{2}$ & $\left(3.31 a_{1}+3.89 a_{2}\right)^{2}$ & $\left(0.30 a_{1}+0.44 a_{2}\right)^{2}$ & $\left(0.48 a_{1}+0.80 a_{2}\right)^{2}$ \\
{$[18]$} & $\left(1.62 a_{1}+1.72 a_{2}\right)^{2}$ & $\left(3.13 a_{1}+3.67 a_{2}\right)^{2}$ & $\left(0.372 a_{1}+0.338 a_{2}\right)^{2}$ & $\left(0.686 a_{1}+0.732 a_{2}\right)^{2}$ \\
\hline
\end{tabular}

In Table 3 we show the values of branching fractions obtained in this work for two different set of the Wilson coefficients $\left(a_{1}=0.93, a_{2}=-0.27\right.$ and $\left.a_{1}=1.14, a_{2}=-0.20\right)$ and compare them with other theoretical approaches.

Table 3. Branching ratios (in \%) of nonleptonic $B_{c}$ decays obtained in this work for two different set of the Wilson coefficients.

\begin{tabular}{|c|c|c|c|c|c|c|c|c|c|}
\hline Mode & $\begin{array}{c}a_{1}=+0.93 \\
a_{2}=-0.27\end{array}$ & $\begin{array}{c}a_{1}=+1.14 \\
a_{2}=-0.20\end{array}$ & {$[3]$} & {$[5]$} & {$[6]$} & {$[9]$} & {$[10]$} & {$[17]$} & {$[18]$} \\
\hline$B_{c} \rightarrow J / \psi D_{s}^{+}$ & 0.10 & 0.22 & 0.34 & 0.34 & 0.17 & 0.14 & 0.81 & 0.12 & 0.15 \\
$B_{c} \rightarrow J / \psi D_{s}^{*+}$ & 0.41 & 0.78 & 0.75 & 0.59 & 0.67 & 0.41 & 2.05 & 0.55 & 0.62 \\
\hline$B_{c} \rightarrow J / \psi D^{+}$ & 0.0035 & 0.0074 & 0.013 & 0.013 & 0.009 & 0.0055 & 0.028 & 0.0044 & 0.009 \\
$B_{c} \rightarrow J / \psi D^{*+}$ & 0.017 & 0.031 & 0.023 & 0.019 & 0.028 & 0.018 & 0.067 & 0.010 & 0.028 \\
\hline
\end{tabular}


Table 4. Comparison of the results for the ratios of nonleptonic branching fractions of $B_{c}$ meson

\begin{tabular}{c|c|c|c|r}
\hline \hline$R_{D_{s}^{*} / D_{s}}$ & $R_{D^{*} / D}$ & $R_{D^{*} / D_{s}^{*}}$ & $R_{D / D_{s}}$ & Ref. \\
\hline $2.8_{-0.9}^{+1.2}$ & & & & ATLAS [1] \\
$2.37 \pm 0.57$ & & & & LHCb [2] \\
\hline $4.1 \pm 0.80$ & $4.86 \pm 0.97$ & $0.041 \pm 0.008$ & $0.035 \pm 0.007$ & CCQM \\
2.9 & 1.77 & 0.031 & 0.038 & RCQM [3] \\
1.7 & 1.46 & 0.032 & 0.038 & QCD PM [5] \\
3.9 & 3.11 & 0.042 & 0.053 & QCD SR [6] \\
$3.01 \pm 1.23$ & 3.27 & 0.044 & 0.039 & LFQM [9] \\
$2.54_{-0.21}^{+0.07}$ & 2.39 & 0.033 & 0.035 & pQCD [10] \\
\hline \hline
\end{tabular}

Finally, we compare our results for ratios of nonleptonic branching fractions of $B_{c}$ meson with available experimental data and the results obtained in other approaches in Table 4, where

$$
R_{D_{1 q}^{(*)} / D_{2 q}^{(*)}}=\frac{B\left(B_{c}^{+} \rightarrow J / \psi D_{1 q}^{(*)+}\right)}{B\left(B_{c}^{+} \rightarrow J / \psi D_{2 q}^{(*)+}\right)} .
$$

\section{Acknowledgment}

Author A. Issadykov is grateful for the support by the JINR, grant number 17-302-03.

\section{References}

[1] G. Aad et al. [ATLAS Collaboration], Eur. Phys. J. C 76 (2016) no.1, 4 [arXiv:1507.07099 [hep-ex]].

[2] R. Aaij et al. [LHCb Collaboration], Phys. Rev. D 87 (2013) no.11, 112012 Addendum: [Phys. Rev. D 89 (2014) no.1, 019901] [arXiv:1304.4530 [hep-ex]].

[3] M. A. Ivanov, J. G. Körner and P. Santorelli, Phys. Rev. D 73 (2006) 054024 [hep-ph/0602050].

[4] C. H. Chang and Y. Q. Chen, Phys. Rev. D 49, 3399 (1994).

[5] P. Colangelo and F. De Fazio, Phys. Rev. D 61, 034012 (2000) [hep-ph/9909423].

[6] V. V. Kiselev, hep-ph/0211021.

[7] D. Ebert, R. N. Faustov and V. O. Galkin, Phys. Rev. D 68, 094020 (2003) [hep-ph/0306306].

[8] A. K. Likhoded and A. V. Luchinsky, Phys. Rev. D 81, 014015 (2010) [arXiv:0910.3089 [hep$\mathrm{ph}]$.

[9] H. W. Ke, T. Liu and X. Q. Li, Phys. Rev. D 89, no. 1, 017501 (2014) [arXiv:1307.5925 [hep$\mathrm{ph}]]$.

[10] Z. Rui and Z. T. Zou, Phys. Rev. D 90, no. 11, 114030 (2014) [arXiv:1407.5550 [hep-ph]].

[11] S. Kar, P. C. Dash, M. Priyadarsini, S. Naimuddin and N. Barik, Phys. Rev. D 88, no. 9, 094014 (2013). 
[12] R. Zhu, Y. Ma, X. L. Han and Z. J. Xiao, Phys. Rev. D 95, no. 9, 094012 (2017) [arXiv:1703.03875 [hep-ph]].

[13] G. Buchalla, A. J. Buras, M. E. Lautenbacher, Rev. Mod. Phys. 68, 1125-1144 (1996). [hep$\mathrm{ph} / 9512380]$.

[14] S. Dubnicka, A. Z. Dubnickova, A. Issadykov, M. A. Ivanov and A. Liptaj, Phys. Rev. D 96 (2017) no.7, 076017 doi:10.1103/PhysRevD.96.076017 [arXiv:1708.09607 [hep-ph]].

[15] M. A. Ivanov, J. G. Körner and P. Santorelli, Phys. Rev. D 63 (2001) 074010 [hep-ph/0007169].

[16] A. Issadykov, M. A. Ivanov and G. Nurbakova, EPJ Web Conf. 158 (2017) 03002. doi:10.1051/epjconf/201715803002

[17] A. Y. Anisimov, P. Y. Kulikov, I. M. Narodetsky and K. A. Ter-Martirosian, Phys. Atom. Nucl. 62 (1999) 1739 [Yad. Fiz. 62 (1999) 1868] [hep-ph/9809249].

[18] A. Abd El-Hady, J. H. Munoz and J. P. Vary, Phys. Rev. D 62 (2000) 014019 doi:10.1103/PhysRevD.62.014019 [hep-ph/9909406]. 